\title{
On Angular Limits of Normal Meromorphic Functions: A Geometric Aspect
}

\author{
Zarko Pavicevic \\ Faculty of Science, University of Montenegro, 81000 Podgorica, Montenegro \\ Correspondence should be addressed to Zarko Pavicevic; zarkop@ac.me
}

Received 27 August 2013; Accepted 11 November 2013; Published 20 January 2014

Academic Editor: Yan Xu

Copyright (C) 2014 Zarko Pavicevic. This is an open access article distributed under the Creative Commons Attribution License, which permits unrestricted use, distribution, and reproduction in any medium, provided the original work is properly cited.

We will prove the assertions which give necessary and sufficient conditions for a normal meromorphic function on the open unit disk to have an angular limit. The results obtained show that the conditions from the classical Lindelöf theorem, as well as the theorems of Lehto and Virtanen and Bagemihl and Seidel, concerning angular limit values of meromorphic functions, can be weakened.

\section{Introduction and Preliminaries}

Let $\mathbb{D}=\{z \in \mathbb{C}:|z|<1\}$ be the open unit disk in the complex plane $\mathbb{C}$, with the boundary $\Gamma=\{z \in \mathbb{C}:|z|=1\}$, and let $\Omega=\overline{\mathbb{C}}=\mathbb{C} \cup\{\infty\}$ be the Riemann sphere. For any $w \in \mathbb{D}$ and a function $f: \mathbb{D} \rightarrow \Omega$, let $f_{w}=f \circ \varphi_{w}$, where $\varphi_{w}(z)=(z+w) /(1+\bar{w} z)(z \in \mathbb{C})$ is a Möbius map. Further, the pseudohyperbolic distance $d_{\mathrm{ph}}$ on $\mathbb{D}$ is given by

$$
d_{\mathrm{ph}}\left(z_{1}, z_{2}\right)=\left|\frac{z_{1}-z_{2}}{1-z_{1} \overline{z_{2}}}\right|, \quad z_{1}, z_{2} \in \mathbb{D} .
$$

The function $d_{h}$ defined on $\mathbb{D}$ as

$$
d_{h}\left(z_{1}, z_{2}\right)=\ln \frac{1+d_{\mathrm{ph}}\left(z_{1}, z_{2}\right)}{1-d_{\mathrm{ph}}\left(z_{1}, z_{2}\right)}, \quad z_{1}, z_{2} \in \mathbb{D},
$$

is the hyperbolic metric on $\mathbb{D}$. The chordal metric on the Riemann sphere is defined as

$$
\begin{aligned}
& d_{s}\left(w_{1}, w_{2}\right) \\
& = \begin{cases}\frac{2\left|w_{1}-w_{2}\right|}{\sqrt{1+\left|w_{1}\right|^{2}} \sqrt{1+\left|w_{2}\right|^{2}}} & \text { if } w_{1}, w_{2} \in \mathbb{C} \\
\frac{2}{\sqrt{1+\left|w_{1}\right|^{2}}} & \text { if } w_{1} \in \mathbb{C}, w_{2}=\infty\end{cases}
\end{aligned}
$$

All the convergence in this paper will be considered with respect to some of the aforementioned metrics. Since the convergence with respect to the hyperbolic and pseudohyperbolic metrics on the disk $\mathbb{D}$ is equivalent, in our proofs we will use one of these metrics that "simplifies" the related proof.

For a fixed $w \in \mathbb{D}$, the set $\mathbb{D}_{\mathrm{ph}}(w, r)$ defined as

$$
\mathbb{D}_{\mathrm{ph}}(w, r)=\left\{z \in \mathbb{D}: d_{\mathrm{ph}}(z, w)<r\right\}, \quad 0<r<1,
$$

is called the pseudohyperbolic disk with the pseudohyperbolic center $w$ and the pseudohyperbolic radius $r$. Notice that for $w=0, \mathbb{D}_{\mathrm{ph}}(0, r)=\{z:|z|<r\}:=\mathbb{D}(0, r), 0<r<1$. Similarly, for a fixed $w \in \mathbb{D}$, the set $\mathbb{D}_{h}(w, r)$ defined as

$$
\mathbb{D}_{h}(w, r)=\left\{z \in \mathbb{D}: d_{h}(z, w)<r\right\}, \quad 0<r<1,
$$

is called the hyperbolic disk with the center $w$ and the hyperbolic radius $r$.

For simplicity, here as always in the sequel, for an arbitrary nonempty set $K \subset \mathbb{D}$ we write $f_{n} \stackrel{K}{\Rightarrow} f$ if a sequence $\left(f_{n}\right)$ of complex functions defined on the disk $\mathbb{D}$ tends uniformly on $K$ to the function $f$ with respect to the pseudohyperbolic (or hyperbolic) metric $d_{\mathrm{ph}}$ (or $d_{h}$ ) and the chordal metric $d_{s}$ of the Riemann sphere $\Omega$.

Given a set $A \subseteq \mathbb{D}$ so that $\bar{A} \cap \Gamma=\left\{e^{i \theta}\right\}(\bar{A}$ is the closure of $A$ ) and the function $f: \mathbb{D} \rightarrow \overline{\mathbb{C}}$, denote by $C\left(f, A, e^{i \theta}\right)$ the cluster set of the function $f$ at the point $e^{i \theta}$ with respect 
to the set $A$. Namely, $C\left(f, A, e^{i \theta}\right)$ is the set of all points $\omega \in \Omega$ for which there exists a sequence $\left(z_{n}\right)$ in $A$ so that $\left(z_{n}\right) \rightarrow e^{i \theta}$ and $f\left(z_{n}\right) \rightarrow \omega$ as $n \rightarrow \infty$. It is known that $C\left(f, A, e^{i \theta}\right)=$ $\overline{C\left(f, A, e^{i \theta}\right)}$. If

$$
\begin{aligned}
A & =\left\{z: z \in \mathbb{D},\left|\arg \left(e^{i \theta}-z\right)\right|<\alpha, 0<\alpha<\frac{\pi}{2}\right\} \\
& :=\Delta\left(e^{i \theta}, \alpha\right)
\end{aligned}
$$

is a Stolz angle of the disk $\mathbb{D}$ with the vertex at the point $e^{i \theta}$, then the cluster set $C\left(f, \Delta\left(e^{i \theta}, \alpha\right), e^{i \theta}\right)$ is the limit value of the function $f$ along the angle $\Delta\left(e^{i \theta}\right)$. If for each $\alpha$ with $0<\alpha<$ $\pi / 2$ we have $C\left(f, \Delta\left(e^{i \theta}, \alpha\right), e^{i \theta}\right)=\{\omega\}$ for some $\omega \in \Omega$, then $e^{i \theta}$ is said to be a Fatou point of the function $f$ and $\omega \in \Omega$ is its angular limit value.

Here, as always in the sequel, $\gamma$ will denote a Jordan arc that ends at a point $e^{i \theta}$. If $C\left(f, \gamma, e^{i \theta}\right)=\{\omega\}$ for some $\omega \in \Omega$ then $\omega$ is said to be an asymptotic value of the function $f$ at the point $e^{i \theta}$ along the curve $\gamma$. The classical Lindelöf theorem on boundary values of holomorphic functions asserts that if a bounded analytic function on the disk $\mathbb{D}$ has an asymptotic value $\omega, \omega \in \Omega$, at a point $e^{i \theta}$, then $\omega$ is its angular limit value and $e^{i \theta}$ is a Fatou point of the function $f[1]$.

Seidel [2] and Seidel and Walsh [3] investigated the boundary properties of bounded analytic functions and analytic functions that omit two complex values via the convergence of their Heine's sequences. They proved that the results of Lindelöf's theorem are also valid for univalent analytic functions.

In [4], Lehto and Virtanen extended Lindelöf's result to the class of normal meromorphic functions in $\mathbb{D}$.

A function $f$ meromorphic on the disk $\mathbb{D}$ is said to be normal in $\mathbb{D}$ if the family $\mathscr{F}_{f}:=\left\{f_{w}: f_{w}:=f \circ \varphi_{w}: w \in \mathbb{D}\right\}$ is normal in the disk $\mathbb{D}$ in the sense of Montel, which means that each sequence in $\mathscr{F}_{f}$ contains a subsequence which converges uniformly on each compact subset of $\mathbb{D}$. Namely, Lehto and Virtanen proved that if a normal meromorphic function in the disk $\mathbb{D}$ has an asymptotic value $\omega, \omega \in \Omega$, at a point $e^{i \theta}$, then $\omega$ is its angular limit value and $e^{i \theta}$ is a Fatou point of the function $f[4$, Theorem 2].

In the same paper, in terms of the estimate of growth of the spherical derivative, Lehto and Virtanen established a necessary and sufficient conditions for a function $f$ meromorphic on hyperbolic domains of $f$ to be a normal function. For more information on the properties and applications of normal meromorphic functions in the theory of functions see [5].

In [6], Bagemihl and Seidel examined the existence of angular limits of a function $f$ meromorphic on $\mathbb{D}$ at a point $e^{i \theta}, e^{i \theta} \in \Gamma$, via the existence of its Heine's sequences $\left(f\left(z_{n}\right)\right)$, $\left(z_{n}\right) \subset \mathbb{D}$ so that $z_{n} \rightarrow e^{i \theta}$ as $n \rightarrow \infty$.

In further considerations, the notion of the range $R\left(f, e^{i \theta}\right)$ will be significant, where $f$ is a function defined in $\mathbb{D}$. The range $R\left(f, e^{i \theta}\right)$ is defined as the set of all points $c \in \Omega$ so that there exists a sequence $\left(z_{n}\right) \subset \mathbb{D}$ for which $\lim _{n \rightarrow \infty} z_{n}=e^{i \theta}$ and $f\left(z_{n}\right)=c$ for all $n \in \mathbb{N}$.
Bagemihl and Seidel [6, Theorem 1] showed that if for a normal meromorphic function $f$ on $\mathbb{D}$ there exists a sequence $\left(z_{n}\right) \subset \mathbb{D}$ such that $\lim _{n \rightarrow \infty} z_{n}=e^{i \theta}$,

$$
\sup \left\{d_{h}\left(z_{n}, z_{n+1}\right): n \in \mathbb{N}\right\} \leq M<+\infty
$$

and $\lim _{n \rightarrow \infty} f\left(z_{n}\right)=c$ for some $c \in \Omega$ with $c \notin R\left(f, e^{i \theta}\right)$, then $e^{i \theta}$ is a Fatou point of the function $f$ and $c \in \Omega$ is its angular limit value. In [6, Examples 1 and 2], the authors gave examples of analytic functions that show that the condition of normality for the function $f$ and the condition

$$
\sup \left\{d_{h}\left(z_{n}, z_{n+1}\right): n \in \mathbb{N}\right\} \leq M<+\infty
$$

related to the sequence $\left(z_{n}\right) \subset \mathbb{D}$ with $\lim _{n \rightarrow \infty} z_{n}=e^{i \theta}$ cannot be omitted. Example 4 in [6] of a Blaschke product shows the fact that the condition $c \notin R\left(f, e^{i \theta}\right)$ is necessary in that assertion.

Further boundary properties of meromorphic functions via their behaviour along sequences $\left(z_{n}\right) \subset \mathbb{D}$ with $\lim _{n \rightarrow \infty} z_{n}=e^{i \theta}$ were investigated by Gavrilov (see, e.g., $[5,7,8])$ and Gauthier $([9,10])$.

In this paper, we prove Theorem 6 of Section 3 and Theorems 7-10 of Section 4, which give necessary and sufficient conditions for a normal meromorphic function $f$ in $\mathbb{D}$ at a point $e^{i \theta}$ to have an angular limit in terms of a sequence $\left(f\left(z_{n}\right)\right)$ with $\left(z_{n}\right) \subset \mathbb{D}$ and $\lim _{n \rightarrow \infty} z_{n}=e^{i \theta}$ under the condition $\sup \left\{d_{h}\left(z_{n}, z_{n+1}\right): n \in \mathbb{N}\right\} \leq M<+\infty$. Here it is not supposed that $c \notin R\left(f, e^{i \theta}\right)$. The omission of the condition $c \notin R\left(f, e^{i \theta}\right)$ and the retention of the condition $\sup \left\{d_{h}\left(z_{n}, z_{n+1}\right): n \in \mathbb{N}\right\} \leq M<+\infty$ related to terms of the sequence $\left(z_{n}\right)$ require "good" boundary behaviour of the function $f$ along sufficiently "thick" sets whose elements lie within a "small hyperbolic distance" from the points $z_{n} \in$ $\mathbb{D}, n \in \mathbb{N}$, with $\lim _{n \rightarrow \infty} z_{n}=e^{i \theta}$ and $\sup \left\{d_{h}\left(z_{n}, z_{n+1}\right)\right.$ : $n \in \mathbb{N}\} \leq M<+\infty$. Namely, in Theorem 7 these sets are sufficiently large disks whose pseudohyperbolic (hyperbolic) centers are the terms of the sequence $\left(z_{n}\right)$ (see Figure 1 ); in Theorem 8 , these sets are sufficiently large parts of Jordan's arcs, and in Theorem 10 they are many sequences of points. These theorems give new criteria for the existence of angular limit values of meromorphic functions on $\mathbb{D}$ at points $e^{i \theta}$ of the unit circle $\Gamma$.

In Section 2, we prove Lemmas 1, 3, and 4. These lemmas present auxiliary results for proofs of other assertions in this paper.

\section{Auxiliary Lemmas}

In this section, we consider the uniform convergence of function sequences to constant functions. Related classical results, for example, can be found in [11-13], while the uniform convergence of function sequences via properties of boundary sets of functions were investigated in $[14,15]$; also see [16].

Lemma 1. Let $f: \mathbb{D} \rightarrow \Omega$ be an arbitrary function, let $\left(w_{n}\right) \subset$ $\mathbb{D}$ be a sequence such that $\lim _{n \rightarrow \infty} w_{n}=e^{i \theta}$, and let $G$ be 


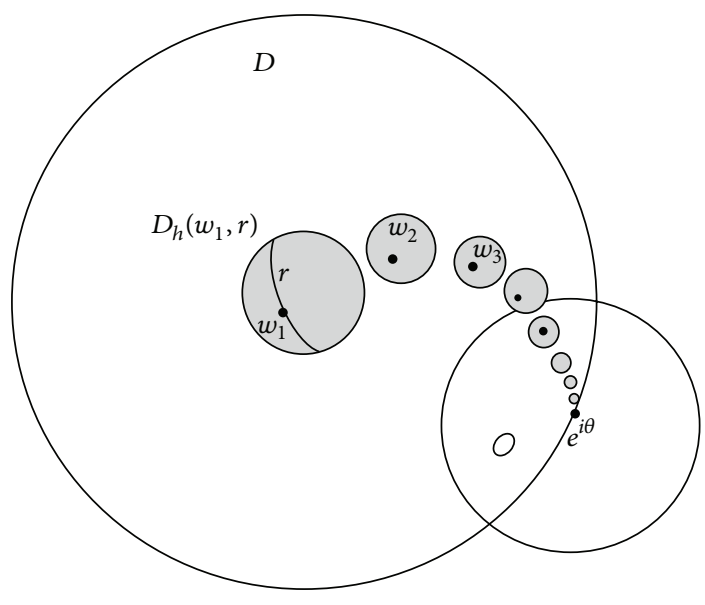

FIGURE 1

an arbitrary nonempty subset of $\mathbb{D}$ with $G \subset \mathbb{D}(0, r), 0<r<1$. Then the following conditions are equivalent:

(i) $f_{n} \stackrel{G}{\Rightarrow} c$ with $c \in \Omega$ and $f_{n}=f \circ \varphi_{w_{n}}, n \in \mathbb{N}$;

(ii) $C\left(f, \cup_{n=1}^{\infty} \varphi_{w_{n}}(G), e^{i \theta}\right)=\{c\}$.

Proof. From the condition $\lim _{n \rightarrow \infty} w_{n}=e^{i \theta}$ it follows that

$$
\left(\overline{\bigcup_{n=1}^{\infty} \varphi_{w_{n}}(G)}\right) \cap \Gamma=\left\{e^{i \theta}\right\}
$$

(i) $\Rightarrow$ (ii). It follows from (i) that for each $\varepsilon>0$ there exists a positive integer $N=N(\varepsilon)$ such that $d_{s}\left(\left(f \circ \varphi_{w_{n}}\right)(z), c\right)<\varepsilon$ for all $n \geq N$ and $z \in G$. This yields that $f\left(\varphi_{w_{n}}(G)\right) \subset\{\omega$ : $\left.\omega \in \Omega, d_{s}(c, \omega)<\varepsilon\right\}$ for all $n \geq N$ and $z \in G$, and thus,

$$
f\left(\bigcup_{n=N}^{\infty} \varphi_{w_{n}}(G)\right) \subset\left\{\omega: \omega \in \Omega, d_{s}(c, \omega)<\varepsilon\right\},
$$

that is,

$$
d_{s}(c, f(z))<\varepsilon, \quad \text { for each } z \in \bigcup_{n=N}^{\infty} \varphi_{w_{n}}(G)
$$

Let $\left(z_{m}\right)$ be a sequence contained in $\cup_{n=1}^{\infty} \varphi_{w_{n}}(G)$ such that $\lim _{m \rightarrow \infty} z_{m}=e^{i \theta}$. We will show that $\lim _{m \rightarrow \infty} f\left(z_{m}\right)=c$. For each $m \in \mathbb{N}$ there is a $n_{m} \in \mathbb{N}$ such that $z_{m} \in \varphi_{w_{n_{m}}}(G)$. From $\lim _{m \rightarrow \infty} z_{m}=e^{i \theta}$ it follows that $\lim _{m \rightarrow \infty} n_{m}=\infty$, and so, for $k=N$ there is a $M=M(N) \in \mathbb{N}$ such that $n_{m} \geq N$ for every $m \geq M$. As $z_{m} \in \varphi_{w_{n_{m}}}(G)$, by the previous argument it follows that $z_{m} \in \cup_{n=N}^{\infty} \varphi_{w_{n}}(G)$ for all $m \geq M$. This together with (11) gives

$$
d_{s}\left(c, f\left(z_{m}\right)\right)<\varepsilon \quad \text { for each } m \geq M .
$$

Hence, for each $\varepsilon>0$ there exists $M \in \mathbb{N}, M=M(N(\varepsilon))=$ $M(\varepsilon)$ such that $d_{s}\left(c, f\left(z_{m}\right)\right)<\varepsilon$ for each $m \geq M$. Therefore, $\lim _{m \rightarrow \infty} f\left(z_{m}\right)=c$ and $C\left(f, \cup_{n=1}^{\infty} \varphi_{w_{n}}(G), e^{i \theta}\right)=\{c\}$. (ii) $\Rightarrow$ (i). Suppose that (i) does not hold. Then we will show that (ii) also is not satisfied. The assumption that (i) is not true implies that there exists $\varepsilon>0$ such that for every $n \in \mathbb{N}$ there exist $m_{n} \in \mathbb{N}$ and $z \in G$ satisfying $d_{s}\left(c, f\left(\varphi_{w_{m_{n}}}(z)\right), c\right) \geq \varepsilon$. Take $u_{n}=\varphi_{w_{m_{n}}}(z), n \in \mathbb{N}$. Then $u_{n} \in \cup_{k=1}^{\infty} \varphi_{w_{k}}(G)$ for each $n \in \mathbb{N}$, and from $\lim _{n \rightarrow \infty} w_{n}=e^{i \theta}$ it follows that $\lim _{n \rightarrow \infty} w_{m_{n}}=e^{i \theta}$, and so, we have $\lim _{n \rightarrow \infty} u_{n}=$ $e^{i \theta}$. Therefore, we obtain $d_{s}\left(f\left(u_{n}\right), c\right) \geq \varepsilon$, whence it follows that there exists a sequence $\left(u_{n}\right)$ contained in $\cup_{n=1}^{\infty} \varphi_{w_{n}}(G)$ such that $\lim _{n \rightarrow \infty} u_{n}=e^{i \theta}$, but the condition $\lim _{n \rightarrow \infty} f\left(u_{n}\right)=c$ is not satisfied. Hence, the condition (ii) is not satisfied, and the proof is completed.

Lemma 2 (Schiff [13, pp. 72-75]). Let $\left(f_{n}\right)$ be a sequence of meromorphic functions $f_{n}: U \rightarrow \Omega$, where $U$ is a domain in complex plane $\mathbb{C}$. If $\left(f_{n}\right)$ forms a normal family of functions on $U$ and there exists a set $E$ with $E \subset U$ which has at least one accumulation point in $U$, such that a sequence $\left(f_{n}(z)\right)$ converges to the limit function $f(z)$ for each $z \in E$, then $f_{n} \stackrel{K}{\Rightarrow} f$ on each compact subset $K$ of $U$.

Lemma 3. Let $\left(f_{n}\right)$ be a sequence of meromorphic functions $f_{n}: U \rightarrow \Omega$, where $U$ is a domain in complex plane $\mathbb{C}$. If $\left(f_{n}\right)$ forms a normal family of functions on $U$ and there exists a set $E$ with $E \subset U$ which has at least one accumulation point in $U$, such that $\lim _{n \rightarrow \infty} f_{n}(z)=c$, with some $c \in \Omega$, for each $z \in E$, then $f_{n} \stackrel{K}{\Rightarrow} f$ on each compact subset $K$ of $U$ and $f(z) \equiv c$ on U.

Proof. By Lemma 2, it follows that $f_{n} \stackrel{G}{\Rightarrow} f$ on each compact subset $G$ of $U$, where $f$ is a meromorphic function in the domain $U$. From the conditions of Lemma 3, it follows that $f(z)=c$ for each $z \in E$. As the set $E$ has an accumulation point in $U$, by the uniqueness theorem for meromorphic functions, it follows that $f(z) \equiv c$ on $U$, and thus, $f_{n} \stackrel{G}{\Rightarrow} c$ on each compact subset $G$ of $U$.

Lemma 4. Let $f: \mathbb{D} \rightarrow \Omega$ be a normal meromorphic function in the disk $\mathbb{D}$, let $\left(w_{n}\right) \subset \mathbb{D}$ be a sequence such that $\lim _{n \rightarrow \infty} w_{n}=e^{i \theta}$, and let $E$ be an arbitrary subset of $\mathbb{D}$ with $E \subset \mathbb{D}(0, r), 0<r<1$, which has at least one accumulation point. Then the following assertions are equivalent:

(i) $C\left(f, \cup_{n=1}^{\infty} \varphi_{w_{n}}(E), e^{i \theta}\right)=\{c\}$ with $c \in \Omega$;

(ii) For each $r>0, C\left(f, \cup_{n=1}^{\infty} \mathbb{D}_{h}\left(w_{n}, r\right), e^{i \theta}\right)=\{c\}$.

Proof. (i) $\Rightarrow$ (ii). By (i) and Lemmas 1 and 3 it follows that $f_{n} \stackrel{G}{\Rightarrow} c$ on each compact subset $G$ of $\mathbb{D}$. Taking $G=$ $\overline{\mathbb{D}\left(0, r^{\prime}\right)}$, with $0<r^{\prime}<1$, then applying Lemma 1 , we find that $C\left(f, \cup_{n=1}^{\infty} \varphi_{w_{n}}\left(\overline{\mathbb{D}\left(0, r^{\prime}\right)}\right), e^{i \theta}\right)=\{c\}$. Since $\varphi_{w_{n}}\left(\overline{\mathbb{D}\left(0, r^{\prime}\right)}\right)=$ $\overline{\mathbb{D}_{\mathrm{ph}}\left(w_{n}, r^{\prime}\right)}$, we have $C\left(f, \cup_{n=1}^{\infty} \varphi_{w_{n}}\left(\overline{\mathbb{D}_{\mathrm{ph}}\left(w_{n}, r^{\prime}\right)}\right), e^{i \theta}\right)=\{c\}$ for each $r^{\prime}$ with $0<r^{\prime}<1$, and therefore, $C\left(f, \overline{\mathbb{D}_{\mathrm{ph}}\left(w_{n}, r^{\prime}\right)}, e^{i \theta}\right)=$ $\{c\}$ for all $r^{\prime}$ with $0<r^{\prime}<1$ and $n \in \mathbb{N}$. Since for each $r^{\prime}$ with $0<r^{\prime}<1$ and $n \in \mathbb{N}$, it holds $\overline{\mathbb{D}_{\mathrm{ph}}\left(w_{n}, r^{\prime}\right)}=\overline{\mathbb{D}_{h}\left(w_{n}, r\right)}$ 
with $r=\ln \left(\left(1+r^{\prime}\right) /\left(1-r^{\prime}\right)\right)$, we have $C\left(f, \overline{\mathbb{D}_{h}\left(w_{n}, r\right)}, e^{i \theta}\right)=\{c\}$ for each $r>0$ and $n \in \mathbb{N}$.

For any given $r>0$, there exists $r_{1}$ with $0<r_{1}<r$ for which $\overline{\mathbb{D}_{h}\left(w_{n}, r_{1}\right)} \subset \mathbb{D}_{h}\left(w_{n}, r\right) \subset \overline{\mathbb{D}_{h}\left(w_{n}, r\right)}$. Then

$$
\begin{aligned}
\{c\} & =C\left(f, \overline{\mathbb{D}_{h}\left(w_{n}, r_{1}\right)}, e^{i \theta}\right) \\
& \subseteq C\left(f, \mathbb{D}_{h}\left(w_{n}, r\right), e^{i \theta}\right) \\
& \subseteq C\left(f, \overline{\mathbb{D}_{h}\left(w_{n}, r\right)}, e^{i \theta}\right)=\{c\},
\end{aligned}
$$

whence we see that $C\left(f, \mathbb{D}_{h}\left(w_{n}, r\right), e^{i \theta}\right)=\{c\}$ for all $n \in \mathbb{N}$. This shows that

$$
C\left(f, \bigcup_{n=1}^{\infty} \mathbb{D}_{h}\left(w_{n}, r\right), e^{i \theta}\right)=\{c\} \quad \text { for each } r>0
$$

as desired.

(ii) $\Rightarrow$ (i). Suppose that $r>0$ and $C\left(f, \cup_{n=1}^{\infty} \mathbb{D}_{h}\left(w_{n}, r\right)\right.$, $\left.e^{i \theta}\right)=\{c\}$. Then

$$
C\left(f, \bigcup_{n=1}^{\infty} \overline{\mathbb{D}_{h}\left(w_{n}, r\right)}, e^{i \theta}\right)=\{c\} \quad \text { for each } r>0
$$

and hence, $C\left(f, \overline{\mathbb{D}_{h}\left(w_{n}, r\right)}, e^{i \theta}\right)=\{c\}$ for all $r>0$ and $n \epsilon$ $\mathbb{N}$. Since a sequence $\left(f_{n}\right)$ defined as $f_{n}=f \circ \varphi_{w_{n}}$ forms a normal family of functions on $\mathbb{D}$, it follows by Lemma 3 that $f_{n} \stackrel{G}{\Rightarrow} c$, with $c \in \Omega$, for each compact subset $G$ of $\mathbb{D}$. Therefore, for each compact subset $K$ of $\mathbb{D}$ which has at least one accumulation point, we have $f_{n} \stackrel{K}{\Rightarrow} c$. Then by Lemma 1 , $C\left(f, \cup_{n=1}^{\infty} \varphi_{w_{n}}(K), e^{i \theta}\right)=\{c\}$, as required.

Lemma 5 (Schiff $[13$, p. 98]). Let $\mathscr{F}$ be a normal family of meromorphic functions $f: U \rightarrow \Omega$, where $U$ is a domain of the complex plane $\mathbb{C}$, and suppose that $f(z) \neq c, c \in \Omega$, for all $f \in \mathscr{F}$ and $z \in U$. Then for every meromorphic function $\varphi$ for which there exists a sequence $\left(f_{n}\right) \subset \mathscr{F}$ such that $f_{n} \stackrel{G}{\Rightarrow} \varphi$ on each compact subset $G$ of $U$ either there holds $\varphi(z) \neq c$ for each $z \in U$, or $\varphi(z) \equiv$ con $U$.

\section{The Main Result}

Theorem 6. Let $f: \mathbb{D} \rightarrow \Omega$ be a normal meromorphic function in $\mathbb{D}$. Then the following assertions are equivalent.

(i) There exists a sequence $\left(w_{n}\right) \quad \subset \quad \mathbb{D}$ such that $\lim _{n \rightarrow \infty} w_{n}=e^{i \theta}$,

$$
\sup \left\{d_{h}\left(w_{n}, w_{n+1}\right): n \in \mathbb{N}\right\} \leq M<+\infty,
$$

and let $E$ be an arbitrary subset of $\mathbb{D}$ with $E \subset \mathbb{D}(0, r)$, $0<r<1$, which has at least one accumulation point for which $C\left(f, \cup_{n=1}^{\infty} \varphi_{w_{n}}(E), e^{i \theta}\right)=\{c\}, c \in \Omega$;

(ii) A point $e^{i \theta}$ is a Fatou point of a function $f$, and $c \in \Omega$ is its angular limit value at $e^{i \theta}$.
Proof. (i) $\Rightarrow$ (ii). From (i) and Lemma 4 it follows that for any $r>0$,

$$
C\left(f, \bigcup_{n=1}^{\infty} \mathbb{D}_{h}\left(w_{n}, r\right), e^{i \theta}\right)=\{c\}
$$

Suppose that $r>M$. Then $\cup_{n=1}^{\infty} \mathbb{D}_{h}\left(w_{n}, r\right)$ is a simply connected domain. If $L_{n}$ denotes the arc of geodesic curve in hyperbolic metric that joins points $w_{n}$ and $w_{n+1}$, then $\gamma=$ $\cup_{n=1}^{\infty} L_{n}$ is a Jordan curve that lies in $\cup_{n=1}^{\infty} \mathbb{D}_{h}\left(w_{n}, r\right)$ and ends at the point $e^{i \theta}$. Therefore, $C\left(f, \gamma, e^{i \theta}\right) \subset C\left(f, \cup_{n=1}^{\infty} \mathbb{D}_{h}\left(w_{n}, r\right)\right.$, $\left.e^{i \theta}\right)=\{c\}$, whence it follows that $c$ is an asymptotic value of a function $f$ at point $e^{i \theta}$. Then by [4, Theorem 2], it follows that $e^{i \theta}$ is a Fatou point of a function $f$, and $c \in \Omega$ is its angular limit.

(ii) $\Rightarrow\left(\right.$ i). Let $\rho_{\theta}=\left[0, e^{i \theta}\right]$ be the radius of the disk $\mathbb{D}$ that joins the center 0 of $\mathbb{D}$ and a point $e^{i \theta}$ on the unit circle $\Gamma$. Then the set $\cup_{w \in \rho_{\theta}} \mathbb{D}_{h}(w, r), r>0$, is a domain bounded by parts of two hypercircles $H_{\theta}^{+}$and $H_{\theta}^{-}$which join points $e^{i \theta}$ and $-e^{i \theta}$ and by arc of the circle $\Gamma_{r}=\{z:|z|=r\}$. Hypercircles $H_{\theta}^{+}$and $H_{\theta}^{-}$form the angle $\alpha=(\pi / 2)-\operatorname{arctg} e^{-r \sqrt{r}}$ with the radius $\rho_{\theta}$ at the point $e^{i \theta}$. Therefore, a domain $\cup_{w \in \rho_{\theta}} \mathbb{D}_{h}(w, r)$ lies in a Stolz angle $\Delta\left(e^{i \theta}, 2 \alpha\right)$. Then assuming that the terms of a sequence $\left(w_{n}\right)$ are points of the radius $\rho_{\theta}$ satisfying $\lim _{n \rightarrow \infty} w_{n}=e^{i \theta}$ and (16) and taking the disk $\mathbb{D}\left(0, r_{1}\right)$ with $0<r_{1}<r$ as the set $E$, we have

$$
\bigcup_{n=1}^{\infty} \varphi_{w_{n}}\left(\mathbb{D}_{h}\left(0, r_{1}\right)\right)=\bigcup_{w \in \rho_{\theta}} \mathbb{D}_{h}(w, r) \subset \Delta\left(e^{i \theta}, \alpha\right) .
$$

Since $e^{i \theta}$ is a Fatou point of a function $f$ and $c \in \Omega$ is its angular limit, we conclude that $C\left(f, \cup_{n=1}^{\infty} \varphi_{w_{n}}(\mathbb{D}(0, r)), e^{i \theta}\right)=$ $\{c\}$, as desired. This completes the proof.

\section{Applications}

Theorem 7. Let $f: \mathbb{D} \rightarrow \Omega$ be a normal meromorphic function in the disk $\mathbb{D}$, and let $\left(w_{n}\right)$ be a sequence contained in $\mathbb{D}$ satisfying $\lim _{n \rightarrow \infty} w_{n}=e^{i \theta}$ and (16). If $C\left(f, \cup_{n=1}^{\infty} \mathbb{D}_{h}\left(w_{n}, r\right), e^{i \theta}\right)=$ $\{c\}, c \in \Omega$, for somer $>0$, then $e^{i \theta}$ is a Fatou point of a function $f$, and $c \in \Omega$ is its angular limit at $e^{i \theta}$.

Proof. The set $\cup_{n=1}^{\infty} \mathbb{D}_{h}\left(w_{n}, r\right)$ is presented in Figure 1. As $\varphi_{w_{n}}^{-1}\left(\mathbb{D}_{h}\left(w_{n}, r\right)\right)=\mathbb{D}\left(0, r^{\prime}\right)$, with $r^{\prime}=\left(e^{r}-1\right) /\left(e^{r}+1\right)$, that is, $\mathbb{D}_{h}\left(w_{n}, r\right)=\varphi_{w_{n}}\left(\mathbb{D}\left(0, r^{\prime}\right)\right)$ for all $n \in \mathbb{N}$, we have

$$
\begin{aligned}
C\left(f, \bigcup_{n=1}^{\infty} \varphi_{w_{n}}\left(\mathbb{D}\left(0, r^{\prime}\right)\right), e^{i \theta}\right) \\
\quad=C\left(f, \bigcup_{n=1}^{\infty} \mathbb{D}_{h}\left(w_{n}, r\right), e^{i \theta}\right)=\{c\},
\end{aligned}
$$

and taking $E=\mathbb{D}\left(0, r^{\prime}\right)$ in Theorem 6, from this theorem it follows that $e^{i \theta}$ is a Fatou point of a function $f$, and $c \in \Omega$ is its angular limit. 
Theorem 8. Let $f: \mathbb{D} \rightarrow \Omega$ be a normal meromorphic function in the disk $\mathbb{D}$, and let $\gamma$ be a Jordan arc contained in $\mathbb{D}$ such that $\gamma \cap \Gamma=\left\{e^{i \theta}\right\}$. Further, let $\left(w_{n}\right) \subset \gamma$ be a sequence satisfying $\lim _{n \rightarrow \infty} w_{n}=e^{i \theta}$ and (16), and let $\gamma_{n}=\gamma \cap \mathbb{D}_{h}\left(w_{n}, r\right)$ with $n \in \mathbb{N}$ and $r>0$. If the equality $C\left(f, \cup_{n=1}^{\infty} \gamma_{n}, e^{i \theta}\right)=\{c\}$ holds for some $c \in \Omega$ and for some $r>0$, then $e^{i \theta}$ is a Fatou point of the function $f$, and $c \in \Omega$ is its angular limit at $e^{i \theta}$.

Proof. From the conditions of the theorem it follows that a sequence $\left(f_{n}\right):=\left(f \circ \varphi_{w_{n}}\right)$ forms a normal family of functions meromorphic on the disk $\mathbb{D}$. This means that $\left(f_{n}\right)$ contains a subsequence $\left(f_{n_{k}}\right)$ that converges uniformly on compact subsets of $\mathbb{D}$ to a function $\psi$ meromorphic on $\mathbb{D}$. For simplicity, in the sequel we will write $\left(f_{n}\right)$ instead of $\left(f_{n_{k}}\right)$, and $w_{k}$ instead of $w_{n_{k}}, k \in \mathbb{N}$. The proof will be deduced using the pseudohyperbolic metric. Then $\mathbb{D}_{\mathrm{ph}}\left(w_{n}, r\right)=\varphi_{w_{n}}(\mathbb{D}(0, r))$ for any fixed $r$ with $0<r<1$. Set $\gamma_{k}=\gamma \cap \mathbb{D}_{\mathrm{ph}}\left(w_{k}, r\right)$ and $\Gamma_{k}=$ $\varphi_{w_{k}}^{-1}\left(\gamma_{k}\right) \subset \mathbb{D}(0, r), k \in \mathbb{N}$. For any fixed $m \in \mathbb{N}$ and each $k \in \mathbb{N}$, let $z_{k}^{(m)} \in \Gamma_{k} \cap\left\{z:|z|=r_{m}\right\}$, with $0<r_{0}<r_{m+1}<r_{m}<r<1$. Points $z_{k}^{(m)}$ may be chosen so that $z_{k}^{(m)} \neq z_{j}^{(i)}$ when $k \neq j$ or $m \neq i$. In such a way for any fixed $m \in \mathbb{N}$, we find a sequence $\left(z_{k}^{(m)}\right)_{k=1}^{\infty}:=\left(z_{k}^{(m)}\right)$. It follows that a sequence $\left(z_{k}^{(m)}\right)$ can be chosen so that $\lim _{k \rightarrow \infty} z_{k}^{(m)}=z^{(m)} \in \overline{\mathbb{D}(0, r)}$ because of $\left(z_{k}^{(m)}\right) \subset \mathbb{D}(0, r)$. We will prove that $\psi\left(z^{(m)}\right)=c$ for each $m \in \mathbb{N}$.

Let $\varepsilon$ be an arbitrary positive number. Then

$$
\begin{aligned}
d_{s}\left(\psi\left(z^{(m)}\right), c\right) \leq & d_{s}\left(\psi\left(z^{(m)}\right), \psi\left(z_{k}^{(m)}\right)\right) \\
& +d_{s}\left(\psi\left(z_{k}^{(m)}\right), f_{k}\left(z_{k}^{(m)}\right)\right) \\
& +d_{s}\left(f_{k}\left(z_{k}^{(m)}\right), c\right) .
\end{aligned}
$$

Since $\psi$ is a function meromorphic on $\mathbb{D}$, then using a standard " $<\varepsilon / 3$ " estimate of each term on the right hand side of (20), we find that $d_{s}\left(\psi\left(z^{(m)}\right), c\right)<\varepsilon$ for any given $\varepsilon>0$, and thus, $\psi\left(z^{(m)}\right)=c$ for each $m \in \mathbb{N}$. Because $\left(z^{(m)}\right) \subset \overline{\mathbb{D}(0, r)}$ and since a sequence $\left(z^{(m)}\right)$ has an accumulation point in $\overline{\mathbb{D}(0, r)}$, by using the uniqueness theorem for meromorphic functions it follows that $\psi(z) \equiv c$ on $\overline{\mathbb{D}(0, r)}$.

From the previous part of the proof we see that every subsequence $\left(f_{k}\right)$ of a sequence $\left(f_{n}\right)$ which uniformly converges on compact subsets of the disk $\mathbb{D}$ converges to a constant $c$. Suppose that there exists a subsequence $\left(f_{k}\right)$ of the sequence $\left(f_{n}\right)$ that does not converge uniformly on compact subsets of the disk $\mathbb{D}$ to a constant $c$. Then there exists $\varepsilon>0$ such that for each $p \in \mathbb{N}$ there is a $k_{p} \in \mathbb{N}$ and a point $z_{k_{p}} \in \overline{\mathbb{D}(0, r)}$ satisfying

$$
d_{s}\left(f_{k_{p}}\left(z_{k_{p}}\right), c\right) \geq \varepsilon .
$$

As a sequence $\left(f_{n}\right)$ forms a normal family of meromorphic functions, it follows that a sequence $\left(f_{k_{p}}\right)$ has a subsequence that converges uniformly on compacts of the disk $\mathbb{D}$. As was previously proved, it follows that this subsequence converges uniformly on compacts of the disk $\mathbb{D}$ to a constant $c$. This shows that (21) does not hold for all $p \in \mathbb{N}$. This contradiction proves our assertion.

Hence, as proved above, a sequence $\left(f_{n}\right)$ satisfies $f_{n} \stackrel{\overline{\mathbb{D}(0, r)}}{\Rightarrow}$ c. Since

$$
\mathbb{D}_{h}\left(w_{n}, r^{\prime}\right)=\varphi_{w_{n}}(\mathbb{D}(0, r)), \quad r^{\prime}=\ln \frac{1+r}{1-r},
$$

this together with Lemma 1 yields $C\left(f, \cup_{n=1}^{\infty} \mathbb{D}_{h}\left(w_{n}, r^{\prime}\right), e^{i \theta}\right)=$ $\{c\}$, and so by Theorem 7 it follows that $c$ is an angular limit of a function $f$ at a point $e^{i \theta}$ which is its Fatou point.

Remark 9. Theorem 8 shows that the condition on the existence of asymptotic values of normal meromorphic function from Theorem 2 in [4] by Lehto and Virtanen can be replaced by a weaker condition on the existence of its boundary value along arbitrarily "small" arcs of a Jordan curve, where the hyperbolic distance between the consecutive arcs is uniformly bounded from above.

Theorem 10. Let $f: \mathbb{D} \rightarrow \Omega$ be a normal meromorphic function in the disk $\mathbb{D}$, and let $\left(w_{n}\right)$ be a sequence in $\mathbb{D}$ satisfying $\lim _{n \rightarrow \infty} w_{n}=e^{i \theta}$ and (16). If for any $r$ with $0<r<1$ there exist countably many sequences $\left(z_{n}^{(m)}\right)_{n=1}^{\infty}=\left(z_{n}^{(m)}\right)$, with $z_{n}^{(m)} \in$ $\Gamma_{n}^{(m)}, \Gamma_{n}^{(m)}=\left\{z: d_{\mathrm{ph}}\left(z, w_{n}\right)=r^{(m)}\right\}, 0<r^{(m)}<r^{(m+1)}<r<$ $1, n, m \in \mathbb{N}$, for which $\lim _{n \rightarrow \infty} f\left(z_{n}^{(m)}\right)=c$ for each $m \in \mathbb{N}$, then $e^{i \theta}$ is a Fatou point of a function $f$, and $c$ is its angular limit value.

Proof. Put $\varphi_{w_{n}}^{-1}\left(z_{n}^{(m)}\right)=\omega_{n}^{(m)}$, with $n, m \in \mathbb{N}$. Since $\varphi_{w_{n}}^{-1}\left(\Gamma_{n}^{(m)}\right)=$ $\Gamma\left(0, r^{(m)}\right)=\left\{z:|z|=r^{(m)}\right\}$, we have $\omega_{n}^{m} \in \Gamma\left(0, r^{m}\right)$ with $n \in \mathbb{N}$ and any fixed $m \in \mathbb{N}$. Each of sequences $\left(\omega_{n}^{(m)}\right)_{n=1}^{\infty}, m \in \mathbb{N}$, has a subsequence which tends to a limit value $z^{(m)}$ which lies on the circle $\Gamma\left(0, r^{(m)}\right)$. This shows that $z^{(m)} \neq z^{(m+1)}$ and $z^{(m)} \in \mathbb{D}(0, r)$, with $m \in \mathbb{N}$ and $0<r<1$.

Since a function sequence $\left(f \circ \varphi_{w_{n}}\right):=\left(f_{n}\right)$ forms a normal family of meromorphic functions on the disk $\mathbb{D}$, it follows that $\left(f_{n}\right)$ has a subsequence $\left(f_{n_{k}}\right)$ that converges uniformly on compact subsets of the disk $\mathbb{D}$ to a meromorphic function $\psi$. The remainder of the proof is the same as that of Theorem 8 , where terms of a sequence $\left(z^{(m)}\right)$ and a function $\psi$ have the same notations and roles as the corresponding notions related to the proof of Theorem 8 .

Using the results obtained, we can prove Theorem 1 in [6, p. 4] by Bagemihl and Seidel.

Theorem 11 (Bagemihl and Seidel [6, Theorem 1]). Let $f$ : $\mathbb{D} \rightarrow \Omega$ be a normal meromorphic function in the disk $\mathbb{D}$, and let $\left(w_{n}\right)$ be a sequence in $\mathbb{D}$ satisfying $\lim _{n \rightarrow \infty} w_{n}=e^{i \theta}$, (16) and $\lim _{n \rightarrow \infty} f\left(w_{n}\right)=c$ with $c \notin R\left(f, e^{i \theta}\right)$. Then $e^{i \theta}$ is a Fatou point of a function $f$, and $c$ is its angular limit value.

Proof. We use the same notations as those in the proof of Theorem 8. By the condition $c \notin R\left(f, e^{i \theta}\right)$ it follows that $f(z) \neq c$ on $O=\left\{z:\left|z-e^{i \theta}\right|<r\right\} \cap \mathbb{D}$ for some $r$ with $0<r<1$ (see Figure 1). This shows that, for each term of 
a subsequence $\left(f_{k}\right)$ with $k>K$ of the sequence $\left(f_{n}\right)$ such that $\left(f \circ \varphi_{w_{n}}\right)=\left(f_{n}\right)$ which converges uniformly on compacts of the disk $\mathbb{D}$ to a meromorphic function $\varphi(z)$, there holds $f_{k}(z) \neq c$ for all $z \in \mathbb{D}(0, r)$. Since $\varphi(0)=\lim _{k \rightarrow \infty} f_{k}(0)=$ $\lim _{k \rightarrow \infty} f\left(w_{k}\right)=c$, it follows from Lemma 5 that $f_{k} \stackrel{G}{\Rightarrow} c$ on each compact subset $G$ of $\mathbb{D}$. Further, it follows that $f_{n} \stackrel{G}{\Rightarrow} c$ on each compact subset $G$ of $\mathbb{D}$ (see proof of Theorem 8). This together with Lemma 1 yields $C\left(f, \cup_{n=1}^{\infty} \varphi_{w_{n}}(G), e^{i \theta}\right)=\{c\}$, and so by Theorem 6 , we conclude that $c$ is an angular limit value of a function $f$ at a point $e^{i \theta}$, and $e^{i \theta}$ is its Fatou point. This completes the proof.

\section{Conflict of Interests}

The author declares that there is no conflict of interests regarding the publication of this paper.

\section{References}

[1] E. Lindelöf, "Surun principe général d’analyse et ses applications it la theorie de la representationconforme," Acta Soc. Sci. Fenn, vol. 46, no. 4, pp. 1-35, 1915.

[2] W. Seidel, "On the cluster values of analytic functions," Transactions of the American Mathematical Society, vol. 34, pp. 1-21, 1932.

[3] W. Seidel and J. L. Walsh, "On the derivatives of functions analytic in the unit circle and their radii of univalence and of p-valence," Transactions of the American Mathematical Society, vol. 52, pp. 125-216, 1942.

[4] O. Lehto and K. I. Virtanen, "Boundary behaviour and normal meromorphic functions," Acta Mathematica, vol. 97, pp. 47-65, 1957.

[5] A. Lohwater, "The boundary behavior of analytic function," Itogi Nauki i Tekhniki, vol. 10, pp. 99-259, 1973 (Russian).

[6] F. Bagemihl and W. Seidel, Sequential and Continuous Limits of Meromorphic Functions, vol. 280 of Annales Academiae scientiarum Fennicae Series A I, 1960.

[7] V. I. Gavrilov, "On the distribution of values of functions meromorphic in the unit circle, which are not normal," Matematicheskii Sbornik, vol. 67, pp. 408-427, 1965.

[8] V. I. Gavrilov, "Behavior along chords of meromorphic functions in the unit disk," Doklady Akademii Nauk SSSR, vol. 216, pp. 21-23, 1974.

[9] P. Gauthier, "A criterion for normalcy," Nagoya Mathematical Journal, vol. 32, pp. 277-282, 1968.

[10] P. M. Gauthier, "Cercles de remplissage and asymptotic behaviour along circuitous paths," Canadian Journal of Mathematics, vol. 22, pp. 389-393, 1970.

[11] G. M. Goluzin, Geometričeskaya teoriya funkciŭ kompleksnogo peremennogo, Gosudarstv. Izdat. Tehn.-Teor. Lit., MoscowLeningrad, 1952.

[12] P. Montel, Lecons sur les Familles Normales de Fonctions Analytiques et Leurs Applications, Gauthier- Villars, Paris, France, 1927.

[13] J. L. Schiff, Normal Families, Universitext, Springer, New York, NY, USA, 1993.

[14] A. P. Devyatkov and V. I. Kruglikov, "Removing singularities of sequences of analytic functions," Doklady Akademii Nauk, vol.
406, no. 5, pp. 90-91, 2006, Doklady Akademii Nauk, 406, No. 5, 591-592.

[15] V. I. Kruglikov, "Cluster sets of a sequence of functions," Doklady Akademii Nauk, vol. 357, no. 1, pp. 16-18, 1997.

[16] A. P. Devyatkov, Limit sets and boundary properties of removable singularities of sequences of functions [Dissertation], Tyumen, Russia, 2008 (Russian). 


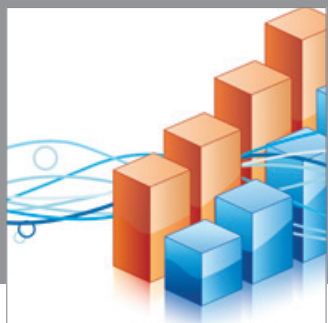

Advances in

Operations Research

mansans

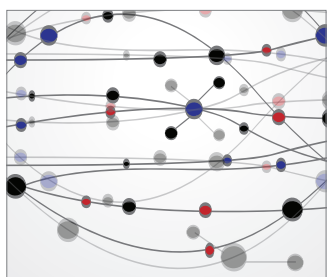

The Scientific World Journal
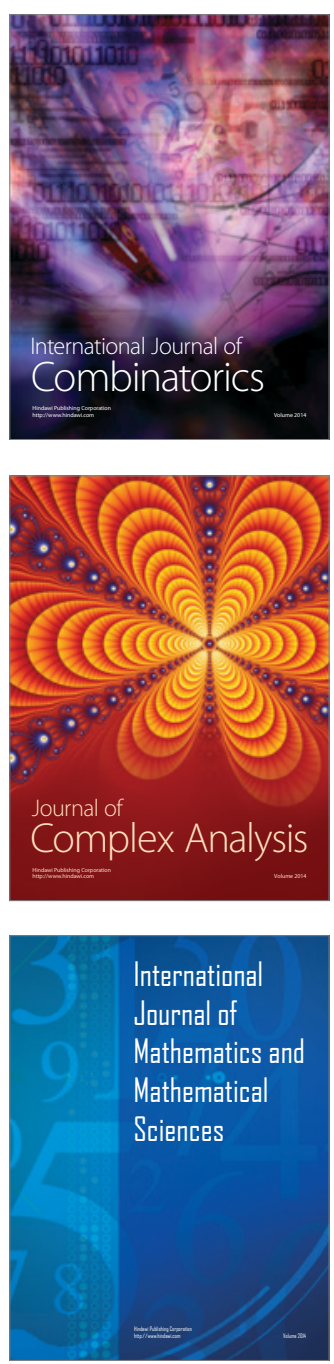
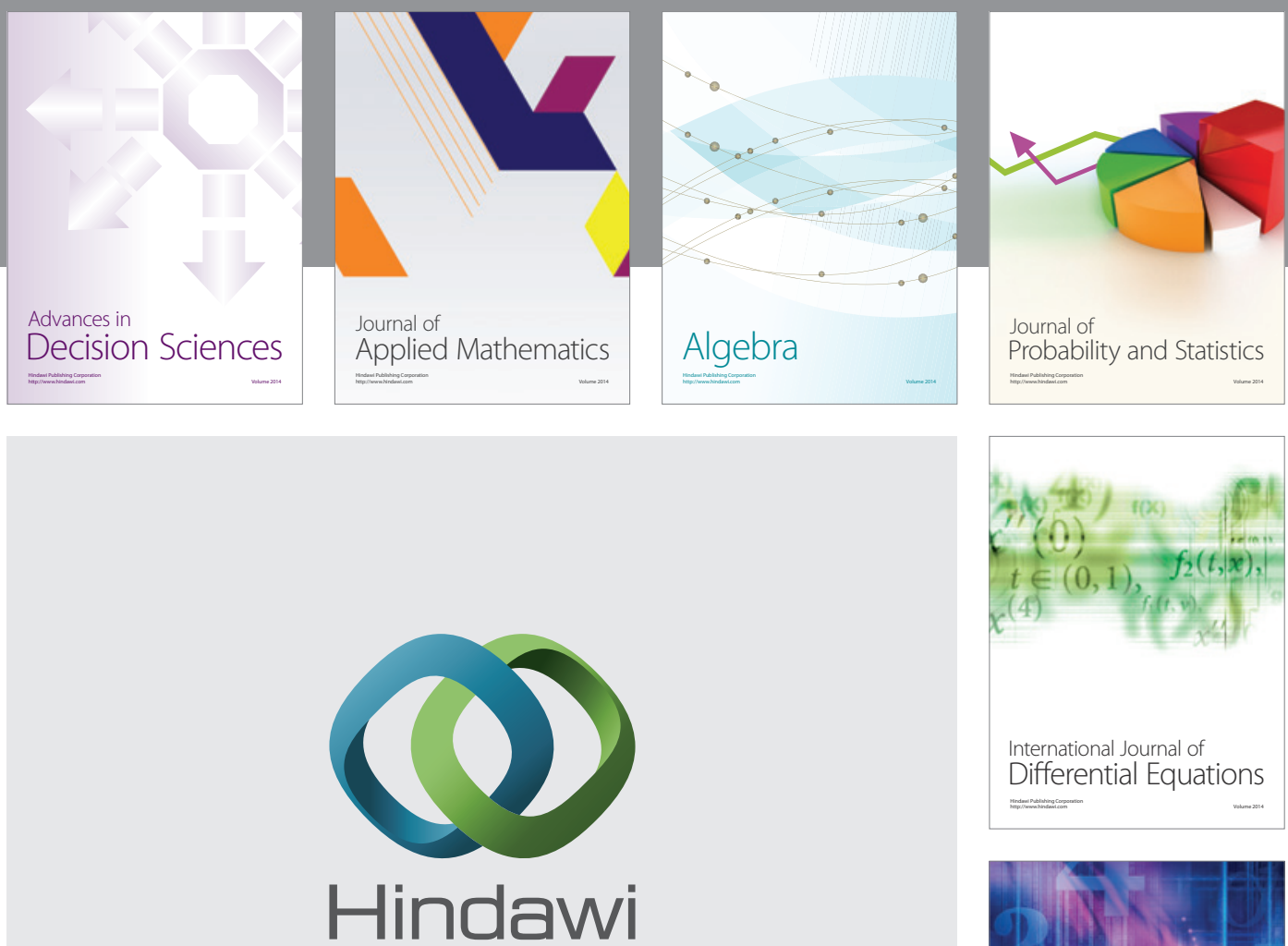

Submit your manuscripts at http://www.hindawi.com
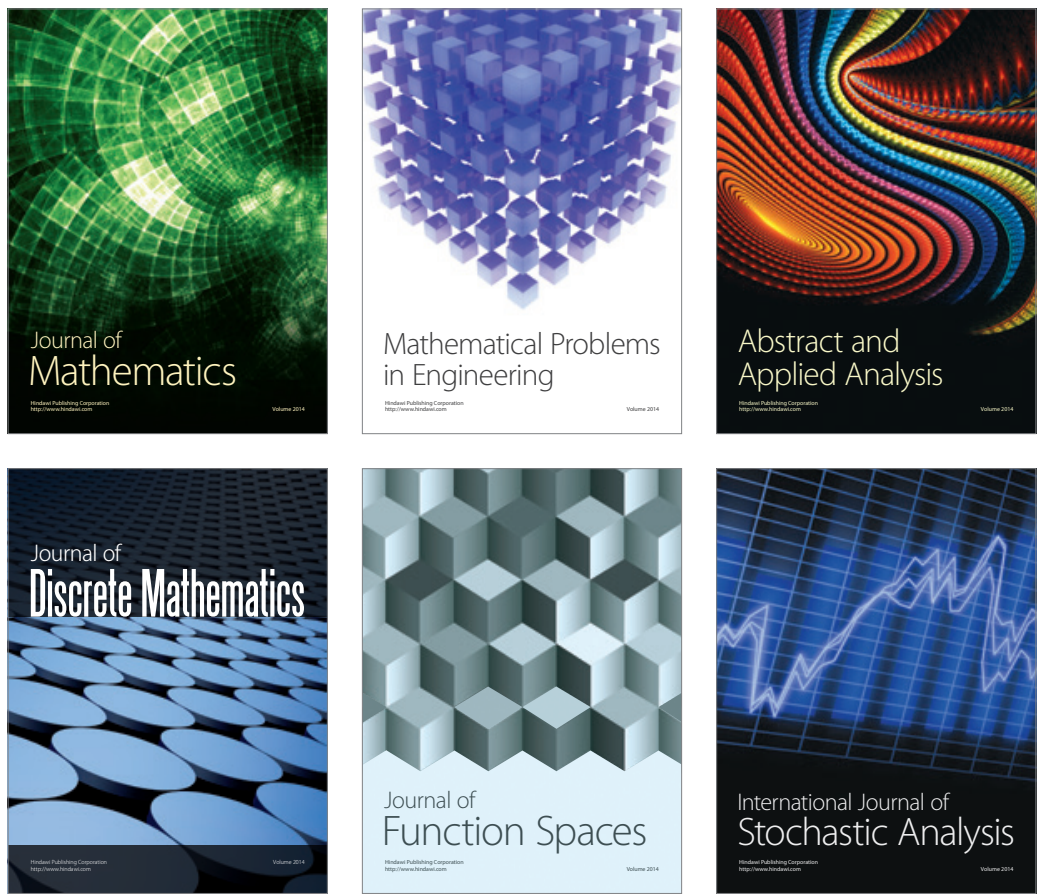

Journal of

Function Spaces

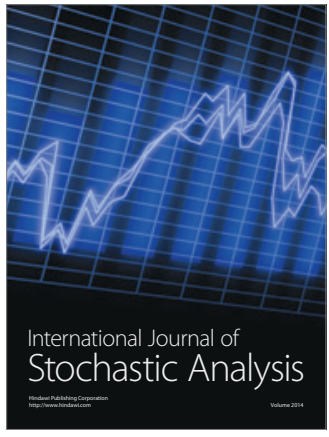

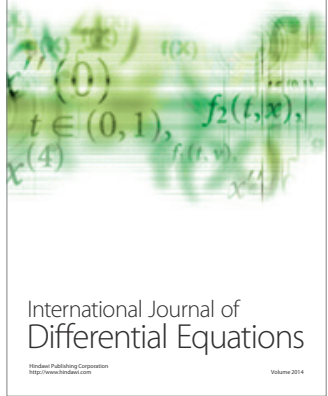
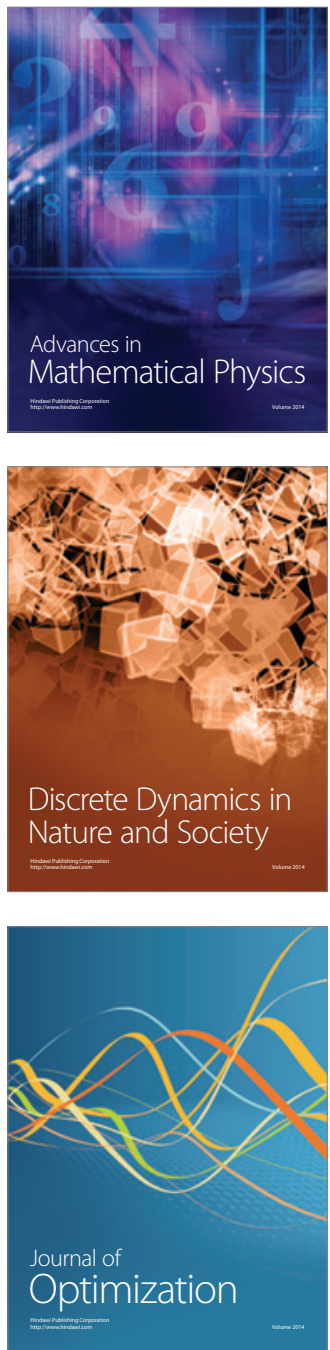